\title{
The role of the visual arts in enhancing the learning process
}

\section{Christopher W. Tyler * and Lora T. Likova}

Smith-Kettlewell Brain Imaging Center, The Smith-Kettlewell Eye Research Institute, San Francisco, CA, USA

\section{Edited by:}

Idan Segev, The Hebrew University of Jerusalem, Israel

\section{Reviewed by:}

Antoni Rodriguez-Fornells, University

of Barcelona, Spain

Lutz Jäncke, University of Zurich,

Switzerland

\section{*Correspondence:}

Christopher W. Tyler, Smith-Kettlewell

Brain Imaging Center, The

Smith-Kettlewell Eye Research

Institute, 2318 Fillmore Street, San

Francisco, CA 94115, USA.

e-mail:cwt@ski.org
With all the wealth of scientific activities, there remains a certain stigma associated with careers in science, as a result of the inevitable concentration on narrow specializations that are inaccessible to general understanding. Enhancement of the process of scientific learning remains a challenge, particularly in the school setting. While direct explanation seems the best approach to expedite learning any specific subject, it is well known that the ability to deeply absorb facts and concepts is greatly enhanced by placing them in a broader context of relevance to the issues of everyday life and to the larger goals of improvement of the quality of life and advancement to a more evolved society as a whole. If the sciences can be associated with areas of artistic endeavor, they may be viewed as more accessible and favorable topics of study. There is consequently an urgent need for research in the relationship between learning and experience in the arts because both art education and scientific literacy remain at an inadequate level even in economically advanced countries. The focus of this review is the concept that inspiration is an integral aspect of the artistic experience, both for the artist and for the viewer of the artwork. As an integrative response, inspiration involves not only higher cortical circuitry but its integration with the deep brain structures such as limbic system and medial frontal structures, which are understood to mediate the experience of emotions, motivational rewards, and the appreciation of the esthetic values of the impinging stimuli. In this sense, inspiration can turn almost any occupation in life into an avocation, a source of satisfaction in achieving life goals. Conversely, when inspiration is lacking, the motivation to learn, adapt, and prosper is impeded. Thus, inspiration may be viewed as a potent aspect of human experience in linking art and science.

Keywords: art, learning, neuroscience, limbic system, inspiration

\begin{abstract}
"How can we develop techniques in learning to engage the emotions? Learning should not be driven by fear but by desire. Why are the arts considered separate from the sciences? What were the first arts? How did the scientific enquiring mind arise?"
\end{abstract}

\section{d'Amboise (2008)}

To a significant extent, our future will be determined by how we deal with the unprecedented explosion of knowledge with which we are presently confronted. In a world of hyper technological advancement, there tends to be an intense focus on the technical and scientific aspects of the world around us, with a consequent neglect of other aspects of life that can enhance the learning of complex material, social skills, and overall quality of life. Although more information has been generated in the past century than in all of human history before it, the gulf between science and the arts has grown ever greater, and students are identified as being of one type or the other early on, creating self-fulfilling prophesies of their life trajectories. Even with all the wealth of scientific activities, there remains a certain stigma associated with careers in science, as a result of the inevitable concentration on narrow specializations that are inaccessible to general understanding. This disconnect between art and science may have had unintended consequences. Apart from the danger of creating a generation of scientists who lack an esthetic sense or appreciation of metaphorical expression, and of artists without scientific literacy, opportunities for cross-pollination, and mutual benefits are being squeezed out of contention by the professional demands.

\section{STUDIES OF ARTS, CREATIVITY, AND LEARNING}

Despite the divergence between arts and sciences, a growing body of quantitative research suggests that the learning of science may be enhanced by relationships with the arts. Contemporary research is beginning to explore explicit neuroscientific hypotheses concerning the effects of activities such as, drawing, visual esthetics, and dance observation.

Visual art learning is reliant on a complex system of perceptual, higher cognitive, and motor functions, thus suggesting a shared neural substrate and strong potential for cross-cognitive transfer in learning and creativity. Within just a few weeks, for example, human infants can imitate and action such as sticking out the tongue in response to someone sticking out his tongue at them - how does the infant know just what motor action plans to implement based only on a visual input? Mirror neurons may account for this ability, translating visual input to motor output, underlying a connection between visual arts and movement, and the auditory arts and music. From pre-historical times, visual art 
has been a form of communication deeply imprinted in human nature; the act of experiencing art and esthetic appreciation in the "receiver" also has the power of cross-cognitive effect any time during individual development. Compositional universals have been shown to govern the design of visual artworks across ages and cultures (Arnheim, 1988; Tyler, 1998, 2007; Ramachandran and Hirstein, 1999).

The ability to tolerate ambiguity and uncertainty during the creative process is an important mental trait. The tolerance for ambiguity is also an important attribute in the learning of science in order to deal with the complexities and ambiguities of scientific knowledge. Unlike its popular stereotype, science is replete with ambiguities and contradictions that have to be resolved in order for learning to proceed. Allusive thinking by appearance alone lends intuitive judgment to overly rational thought and can lead to the discovery of meaningful metaphors (Tucker et al., 1982; Smolucha and Smolucha, 1985; Peterson, 1993). This type of thinking could be developed with focused visual education methods and its applicability shown in a variety of academic disciplines.

In terms of accessible art practice, prior research on neurological patients has shown a conceptual link between drawing and language (Gainotti et al., 1983; Swindell et al., 1988; Kirk and Kertesz, 1989), and these researchers hypothesized that drawing may access the semantic system in a manner that improves cognitive access. Studies exploring the issue of mechanisms shared between different cognitive modalities revealed mechanisms that are used to process spatial representations in the visual modality, are shared with other modalities, such as the processing of pitch in music (Douglas and Bilkey, 2007). These findings have implications not only for scientific learning, but also for learning, pedagogical principles, and general social and educational policies.

\section{THE NEED FOR LEARNING ENHANCEMENT}

The enhancement of learning remains a challenge, particularly in the school setting. While direct explanation seems the best approach to teaching any specific subject on the curriculum, it is well known that the ability to absorb reams of facts and concepts is greatly enhanced by placing them in a broader context of relevance to both the issues of the quality everyday life and the larger goals of human advancement to a more evolved status of society as a whole. It is these larger goals that evoke the need for research in the relationship between learning and experience in the arts. The need is urgent because arts education and scientific literacy remain at a low level in the U.S. and educational interventions are sorely deficient. To the extent that the sciences can be associated with relevant areas of artistic endeavor, they may be viewed as more accessible and more favorable as a topic of study. Moreover, there is an increasing level of neuroscience research that supports the idea of enhancing transfer of learning abilities from the arts to other cognitive domains.

All too often, the arts are marginalized in our schools. In response to this marginalization, educators have sought to justify the arts in terms of their instrumental value in promoting thinking in non-arts subjects considered more important, such as reading or mathematics (Murfee, 1995). However, there has been little convincing research that the study of the arts promotes academic performance or elevates standardized test scores (Winner and Hetland, 2000). Really to understand whether art learning transfers to academic performance, we need first to assess what is actually learned in the arts and then to specify the mechanisms that underlie a transfer hypothesis. Hetland et al. (2007) therefore made a qualitative, ethnographic meta-analysis of the kinds of cognitive skills actually taught in the arts classroom, choosing the visual arts as their point of departure. The goal was to understand what is taught, in order to be able to develop a plausible theoretical transfer hypothesis. Eight "studio habits of mind" were identified as being taught in visual arts classes. Students are taught (1) to observe - to see with acuity; (2) to envision - to generate mental images and imagine; (3) to express - to find their personal voice; (4) to reflect - to think meta-cognitively about their decisions, make critical and evaluative judgments, and justify them; (5) to engage and persist - to work through frustration; (6) to stretch and explore - to take risks, "muck around," and profit from mistakes; and of course (7) to develop craft; and (8) to understand the art world. This work is the first to demonstrate objectively the kinds of thinking skills and working styles taught in arts classes. The group is now investigating the possibility that the skill of envisioning, taught in visual arts classes, may foster geometric reasoning ability.

\section{STUDIES OF INTERSENSORY CONNECTIONS AND THE ARTS}

Neuroimaging studies have revealed that visual arts as well as music engage many aspects of brain function, and involve nearly every neural subsystem identified so far (Zeki, 1999; Solso, 2001; Brown et al., 2006; Cross et al., 2006; Levitin, 2006; Likova, 2010a,b). Could this fact account for claims that arts exercise other part of the brain and improve other cognitive abilities? Experience with the visual arts may be expected to produce similar facilitatory effects through the learning of artistic styles (Hess and Wallsten, 1987), although there is less formal research on the effect of visual art on learning enhancement in general. The visual system is legendary for its ability to analyze the complex interplay among spatial structures in 2D and 3D space. These powerful analytic capabilities are far in advance of what can be achieved by even the most sophisticated computer algorithms, but they are central to any achievement in visual arts (Kubovy, 1986; Gombrich, 1994, 2000; Tyler, 1998; Ramachandran and Hirstein, 1999; Livingstone, 2002). Indeed, neuroscience studies have begun to develop important techniques for the study of the neural circuitry mediating the appreciation of esthetic qualities (Zeki, 2001, 2004; Kawabata and Zeki, 2004; Tononi, 2004). Brain imaging studies have identified the cortical substrates for the encoding of a variety of art-related properties, from primary figure/ground categorization (Likova and Tyler, 2008), and long-range symmetry properties (Tyler, 1994; Norcia et al., 2002; Sasaki et al., 2005; Tyler et al., 2005), through facial expressions (Kanwisher et al., 1997; Zaidel and Cohen, 2005; Chen et al., 2006) to dynamic athletic performances such as dance (Brown et al., 2006; Cross et al., 2006; Brown and Parsons, 2008; Likova, 2010a, 2012a,b). Such experience with the complex structures utilized in the visual arts is likely to make an important contribution to the enhancement of learning in all fields of endeavor. 
The analysis of such complex spatial and dynamic spatial structures is one of the key aspects underlying the creativity of advanced thinking. Creative learning is a key aspect of the human thought processes that crosses many domains of neural functioning (Gardner, 1982; Glover et al., 1989; Csikszentmihalyi, 1997). The role of emotional evaluation in the cognitive processes underlying creativity has been emphasized by Damásio (1994), a theme that he has elaborated into other domains of human endeavor in subsequent work. Indeed, Dietrich (2004) has proposed that there are four basic types of creative learning, each mediated by a distinctive neural circuit. Creativity may arise either from a basis of deliberate control or from spontaneous generation. When the result of deliberate control, the prefrontal cortex instigates the creative process; the spontaneous generation may arise from activation of the temporal cortex. Both processing modes, deliberate and spontaneous, can guide neural computation in structures that contribute emotional content and in those that provide cognitive analysis, yielding the four basic types of creativity. This theoretical framework systematizes the interaction between knowledge and creative thinking, and how the nature of this relationship changes as a function of domain and age.

Defining art as a communicative system that conveys ideas and concepts explaining why it is possible for the same brain structures that supports other cognitive functions such as human language to be involved in arts such as music or drawing. This characterization presuppose millions of years of brain evolution and biological adaptive strategies. As a multidisciplinary communicative system, the arts provide an ideal platform for learning about the pleasure of knowing, which in turn provides the motivational inspiration to explore further, to ask questions, analyze and synthesize, and engage in convergent and divergent thinking.

\section{LEARNING AND ACTIVE INVOLVEMENT IN THE ARTS}

The current expansion of interest in the science of learning motivates exploration of the expanded possibilities of conceptual interrelationships offered by training in the arts. The difficult task of understanding and effectively enhancing learning across disciplines, ages, and cultural specificities is a high priority throughout the world, and may be particularly benefited by training in and even exposure to the arts.

Contemporary research is beginning to explore new neuroscientific hypotheses concerning the effects of learning in activities such as musical performance, drawing, visual esthetics, and dance, on learning in non-artistic domains. Neuroimaging studies have started to reveal that the process of drawing shares cortical substrate with writing, access to the semantic system, memory, naming, imagery, constructional abilities, and the ability to estimate precise spatial relations. Learning in the domain of visual art, in particular, is reliant on a complex system of perceptual, higher cognitive, and motor functions, suggesting a shared neural substrate and strong potential for cross-cognitive transfer in learning and creativity. For instance, case study by Solso (2001) has revealed significant processing differences between the brains of a professional artist and a novice during drawing in the scanner; the comparative analysis of the activation patterns suggests a more effective network of cognitive processing for the brain of the artist. Results consistent with some of these conclusions have also been reported on the basis of differences in alpha rhythm as a function of level of artistic training (Kottlow et al., 2011). Recent neuroimaging studies in our lab have addressed the process of learning to draw by comparing BOLD fMRI brain activity before and after training to draw, and correlating it with the advance in drawing performance. These studies, run in diverse groups of people - from sighted to totally blind from birth, were made possible by a unique Cognitive-Kinesthetic Training Method that Likova developed for learning to draw even under the condition of total blindness. Indeed, in blind subjects who have never had any visual input, training in a spatial drawing skill generates dramatic utilization of occipital lobe resources as early as the primary "visual" cortex for this purely spatial task (despite the complete lack of any visual experience), as well as a reorganization in a network of temporal, parietal, and posterior frontal lobe regions consistent with its multifunctional role (Likova, 2010a,b, 2012a). An additional assessment showed a significant improvement in generic spatial and spatiomotor cognition abilities as well.

Another approach to the neuroanatomical underpinnings of visual art production and appreciation comes from observations of brain damage in established artists have been described (Zaidel and Cohen, 2005), which also provides insight into the relationship between art and other communicative displays by biological organisms, and the role that beauty plays in art. Art should be regarded as a cognitive process in which artists engage the most perplexing issues in present experience and try to find a way of symbolizing them visually so that they can bring coherence to their experience. In consequence, the definition of art is constantly changing in relation to its time. Understanding how we symbolize our experience, how we use symbolic form to organize our psyches, and what are the neuroanatomical corollaries to these processes, will have obvious implications for learning. From pre-historical times, visual art has been a form of communication deeply imprinted in human nature. Compositional universals govern the design of visual artworks across ages and cultures, and the act of art experience and appreciation in the "receiver" also has the power of cross-cognitive effect during any time point in individual development. These findings have implications not only for biomedical sciences, but also for learning, pedagogical principles, and general social and educational policies.

Another key aspect that the arts bring to the mix is the creativity involved in the generation of the art work, which was analyzed into its experiential components by Wallas (1926), involving

(i) preparation by focusing on the domain of problem and prior approaches to its solution,

(ii) incubation by subconscious processes without explicit activity related to the problem,

(iii) intimation that a solution is on its way,

(iv) insight into a novel solution to the problem,

(v) verification and elaboration of the details of the solution.

The Wallas (1926) account is largely cognitive, emphasizing the processes involved in reaching the solution to the problem. He does not specifically address the motivational aspects of how these processes would enhance the learning experience, except in the 
implied rewardingness of the insight (or moment of illumination) of the problem solution. In Wallas's scheme, the preparation stage corresponds to much of the learning required in the educational process, but what is not mentioned is the inspiration that characterizes the motivation for people to take up avocations and hobbies, i.e., the sense of enthusiasm and zeal that some domain of activity is of particular interest or relevance to a person. It is the inspiration, making the preparation stage intrinsically rewarding rather than a painful grind, can make all the difference to the learning experience.

A fine example of the creative moment in science was described by Andrew Feinberg in a keynote lecture on the expanding field of epigenetics (Seay, 2010). When on an architectural visit to Westminster Abbey, he noticed that adjacent to Isaac Newton's grave is a small plaque indicating Paul Dirac's grave (who was awarded the Nobel Prize for advances in the stochastic theory of quantum mechanics). Next to it is Charles Darwin's grave, with no adjacent plaque, but the juxtaposition gave him the epiphany that the modern version of Darwin's theory would be the stochastic variation in epigenetic processes that Feinberg subsequently developed into a major scientific breakthrough. In this case his preparation was many years of scientific research, but it was the foray into the nonscientific architectural tour that gave rise to the novel insight that took his work to the next level.

\section{ARTS, LEARNING, AND INSPIRATION}

Another key aspect of learning that can be facilitated by the arts is the emotional inspiration to be involved in the learning process. Inspiration is an integrative mental function at the intersection of (a) cognitive, (b) emotional, and (c) conative processes. (Conative processes are those goal-directed functions relating to the classic third component of the mind championed by Kant, 1788, and McDougall, 1923, constituting the desire, ambition, and will.) As such, inspiration is an aspect of mental experience that involves not just cortical circuitry but its integration with the limbic system and medial frontal structures that are understood to mediate the experience of emotional desires, motivational rewards, and the appreciation of the integrative esthetic values of the impinging stimuli (Damásio, 1994). This system goes beyond classical concepts of beauty to incorporate the elegance of theoretical concepts, the appreciation of the emotive power of the diverse array of post-modern art installations, the grace and dynamism of athletic performances, the economy and evocativeness of political addresses, the interconnected synergy of natural ecological systems, and innumerable other examples throughout the sphere of our world knowledge. In a sense, inspiration can turn almost any occupation in life into an avocation, a source of satisfaction in achieving life goals. It is when individuals feel themselves part of larger enterprise that they are inspired to learn, to achieve, and to pursue a meaningful career. Conversely, when their job involves performing the same daily drudgery, inspiration is lacking and they lack motivation to learn, adapt, and prosper.

Thus, inspiration is a component of the emotional response to stimuli and actions, when they are perceived as uplifting or emotionally rewarding. As such it should be expected to be mediated by the limbic system and the reward systems of the brain.
An impressive array of neural processing appears to be dedicated to the extraction of reward-related information from environmental stimuli and use of this information in the generation of goal-directed behaviors. In particular, the differential characteristics of activations seen in the dopaminergic mesencephalon, the dorsal striatum, and the orbitofrontal cortex provide distinct examples of the different ways in which reward-related information is processed. Moreover, the differences in activations seen in these three regions demonstrate the different roles they may play in goal-directed behavior (Hollerman et al., 2000). The dopaminergic systems appear to reflect a relatively pure signal of a reward prediction error. The representation of goal-directed behaviors may involve the basal ganglia of the putamen, globus pallidus, and striatum (Acevedo et al., 2011; Paulmann et al., 2011), where different subpopulations neurons differentiate between rewarding and non-rewarding outcomes of behavioral acts and are activated at different stages in the course of goal-directed behaviors, with largely separate populations activated following presentation of conditioned stimuli, preceding reinforcers, and following reinforcers (Apicella et al., 1991; Hollerman et al., 2000). Moreover, unlike the dopamine system, much of the striatal system responds to predicted rewards (Salimpoor et al., 2011). These activations could serve as a component of the neural representation of the appropriate goal-directed behaviors in response to the environmental contingencies associated with desirable goals (Engelmann et al., 2009). Finally, neuronal activations in the orbitofrontal cortex appear to encode the relative motivational significance of different rewards.

Further insights into this reward circuit may be obtained from psychopharmacological studies. In particular, cocaine is known not simply for inducing a sense of reward, but for producing an enhanced (though illusory!) sense of well-being, capability, and quasi-omnipotence. These are the core experiences of inspiration, which can evidently be accessed by this biochemical substitute. Functional imaging studies of focal signal increases for acute cocaine infusion are found in such limbic and basal ganglia structures as the caudate, putamen, basal forebrain, nucleus accumbens, thalamus, hippocampus, and parahippocampal gyrus; in the insular, subcallosal, cingulate, lateral prefrontal, temporal, parietal, and striate/extrastriate cortices; and in the midbrain structure of the ventral tegmentum and the pons (Breiter et al., 1997). Similarly, some of these areas are also implicated in the responses to a romantic image (such subcortical structures as the caudate nucleus, globus pallidus, putamen, lateral thalamus, subthalamic nuclei, and ventral tegmental area; Bartels and Zeki, 2004; Aron et al., 2005; Acevedo et al., 2011). Conversely, acute cocaine infusion produced signal decreases in the temporal pole, medial frontal cortex, and amygdala (Breiter et al., 1997).

As indicated by these studies, activation of the frontal reward network should not be treated as a unitary mental function, since reward in human experience incorporates a diversity of aspects. In particular, it is worth distinguishing four mutually complementary domains of reward - the appetitive, cognitive, social/conative, and inspirational aspects of reward. The first three aspects may be seen as corresponding to the Freudian mental subdivisions of id, ego, and superego functions - i.e., respectively those of hedonic gratification, of the effectiveness of the reward strategies, and 
of the goal-directed achievements of the performer in relation to their place in society (such as course credits for completing the task!). However, the fourth aspect is beyond all these, and relates to how the performer feels about him/herself as a person, more in the realm of Maslow's concept of self-actualization (in the sense of realizing the potential of what one is capable of doing). Specifically, the concept of inspiration focuses on the experiential rather than the functional aspects of this form of achievement-inspiration describes the feeling or awareness of extending one's physical or mental operations beyond the previous capabilities to achieve a new or enhanced capability. It is this expansive or pluripotent sense of the inspirational feeling that make it so significant as a motivational component of human endeavor.

The need for inspiration is something that is well understood by the best teachers, who have the knack of conveying it to their students. However, there is continual pressure to cover specified ranges of exacting material, making it inappropriate for the learning environment to be nothing but entertainment. Thus, the happy medium between sufficient inspiration and the requisite level of proficiency is difficult to achieve, and is made particularly difficult by the wide range of cognitive styles exhibited by the population of learners. There is a strong need to identify the effective motivational styles and the dimensionality of the domain of motivational inspiration, in order to expand the repertoire of strategies for learning enhancement. Moreover, one such form of inspiration is the opportunity to go beyond the predigested material that is presented to develop original insights and contributions to the domain of interest. This form of creativity can be highly motivating to the learner, who feels part of the enterprise of accumulating the knowledge, rather than a passive recipient of the structured material.

\section{RECOMMENDATIONS FOR FUTURE RESEARCH FOR THE ENHANCEMENT OF LEARNING THROUGH ART}

A recent NSF report has assembled recommendations for research strategies for the enhancement of learning through art (Tyler et al., 2009).

\section{STRATEGIC PRINCIPLES}

- Art is fundamentally a communicative medium: the processes of creation and appreciation of art constitutes a special kind of communication; thus future research needs to study both the creators of the art and the consumers (enjoyers) of the artistic products; a focus on one or the other alone would be incomplete.

- Such a dual focus is fundamental to understanding and developing theories of how we learn to create and appreciate art. An adequate theory must account for both the holistic and componential factors that contribute to artistic activities.

- Both art learning and art production involve a complex interplay between multiple sensory-motor and higher cognitive mechanisms. To achieve full understanding of the processes involved in any art, as well as the way they influence learning in other domains, the focus of future investigations should not be restricted within one level of the system, but include consideration of the whole complex of interactions between the levels of learning, art creating, and appreciation.

\section{KEY RESEARCH QUESTIONS}

- How can the dimensionality of the domain be scientifically defined in each of the arts? It is imperative to develop standardized measures and vocabulary in the novel field proposed by the Workshop.

- What are the measurable cognitive and biological underpinnings of learning in specific art forms, such as visual arts, music, dance, theater? How can the relative importance of those learning components be quantified and understood in terms of the neurobiological mechanisms?

- What processing "modules" does art learning share with other cognitive functions, in particular such as those known to be involved in learning and creativity in non-artistic domains?

- What are the implicit benefits and cross-cognitive transfer effects of training and experience in the arts? How can the transformative process of the art experience be studied?

- What is plasticity of the component abilities across the lifespan? What is it about art training that helps people become better artists?

- What is it that the learner is actually learning? What factors do musical, visual art, or dance training impart? What is the link between such training and outcomes in language, social, and cognitive functions?

- Inspiration is an aspect of mental experience that involves not just cortical circuitry but its integration with the limbic system and medial frontal structures that are understood to mediate the experience of emotions, motivational rewards, and the appreciation of the esthetic values of the impinging stimuli. What is the mechanism underlying the role of inspiration in longterm learning? How is inspiration related to the mechanisms of attention and reward?

- Neuroscience studies report a class of "mirror neurons" forming a mirror-matching system that responds similarly when an act is performed by the individual studied or when observing a separate person performing the same act. Does the mirror neural system form the neural substrate of the embodied cognition experienced when viewing a work of art? Can the positive or negative valence of the art-induced form of empathy be harnessed to enhance learning in related fields of endeavor?

- Does learning in an art form always "transfer" to learning in science? (e.g., does the learning of drawing foster the ability to learn geometry, or the learning of music foster the ability to learn language or mathematics?) What factors support or invalidate the operation of such a transfer process?

- When the arts are integrated with other related disciplines in schools, is there evidence that learning in these other disciplines enhanced? Does the answer to this question depend upon the type of learner (e.g., learning disabled; typical)?

- There is a need to evaluate the underlying processes to determine what specific mechanisms for such transfer of learning the brain has developed. What are the main principles of learning transfer and how could they be implemented to effectively enhance educational strategies?

\section{METHODOLOGICAL RECOMMENDATIONS}

- To understand the cross-modal effects of art training, it is necessary to study the basic perceptual processing of the 
artistic objects that give rise to these experiences. The extent to which different key parameters play a role in the artistic experience should be investigated parametrically, and determine how these functions map onto the spectrum of artistic expertise.

- Non-invasive neuroimaging techniques and transcranial magnetic stimulation to generate a reversible blockage of neural activation should be used to answer the questions of learning transfer, enhanced creativity, and enriched esthetic experience.

- Causal network modeling of the information flow amongst cortical regions should be further employed and provide new insights into the neural mechanisms of brain plasticity, which are important for the development of cognitive training strategies.

- Integration of advanced methods must be employed to measure psychophysiological reactions to the artistic experience.

\section{REFERENCES}

Acevedo, B. P., Aron, A., Fisher, H. E., and Brown, L. L. (2011). Neural correlates of long-term intense romantic love. Soc. Cogn. Affect. Neurosci. doi: 10.1093/scan/nsq092

Apicella, P., Ljungberg, T., Scarnati, E., and Schultz, W. (1991). Responses to reward in monkey dorsal and ventral striatum. Exp. Brain Res. 85, 491-500.

Arnheim, R. (1988). The Power of the Center. Berkeley, CA: University of California Press.

Aron, A., Fisher, H., Mashek, D. J., Strong, G., Li, H., and Brown, L. L. (2005). Reward, motivation, and emotion systems associated with early-stage intense romantic love. J. Neurophysiol. 94, 327-337.

Bartels, A., and Zeki, S. (2004). The neural correlates of maternal and romantic love. Neuroimage 21, 1155-1166.

Breiter, H. C., Gollub, R. L., Weisskoff, R. M., Kennedy, D. N., Makris, N., Berke, J. D., Goodman, J. M., Kantor, H. L., Gastfriend, D. R., Riorden, J. P., Mathew, R. T., Rosen, B. R., and Hyman, S. E. (1997). Acute effects of cocaine on human brain activity and emotion. Neuron 19, 591-611.

Brown, S., Martinez, M. J., and Parsons, L. M. (2006). The neural basis of human dance. Cereb. Cortex 16, 1157-1167.

Brown, S., and Parsons, L. M. (2008). The neuroscience of dance. Sci. Am. 7, 78-83.

Chen, C. C., Kao, C., and Tyler, C. W. (2006). Face configuration processing in the human brain: the role of symmetry. Cereb. Cortex 7, 1423-1432.

Cross, E. S., Hamilton, A. F., and Grafton, S. T. (2006). Building a motor simulation de novo: observation of dance by dancers. Neuroimage 31, 1257-1267.

Csikszentmihalyi, M. (1997). Creativity: Flow and the Psychology of Discovery and Invention. New York: Harper Collins.

Damásio, A. (1994). Descartes' Error: Emotion, Reason and the Human Brain. New York: Putnam.

d'Amboise, J. (2008). Remarks at the NSF Workshop on Art, Creativity and Learning. Arlington, VA: National Science Foundation.

Dietrich, A. (2004). The cognitive neuroscience of creativity. Psychon. Bull. Rev. 11, 1011-1026.

Douglas, K. M., and Bilkey, D. K. (2007). Amusia is associated with deficits in spatial processing. Nat. Neurosci. 10, 915-921.

Engelmann, J. B., Damaraju, E., Padmala, S., and Pessoa, L. (2009). Combined effects of attention and motivation on visual task performance: transient and sustained motivational effects. Front. Hum. Neurosci. 3:4, doi: 10.3389/neuro.09.004. 2009

Gainotti, G., Silveri, M. C., Villa, G., and Caltagirone, C. (1983). Drawing objects from memory in aphasia. Brain 106, 613-622.

Gardner, H. (1982). Art, Mind, and Brain: A Cognitive Approach to Creativity. New York: Basic Books.

Glover, J. A., Ronning, R. R., and Reynolds, C. R. (1989). Handbook of Creativity. New York: Plenum Press.

Gombrich, E. H. (1994). The Image and the Eye: Further Studies in the Psychology of Pictorial Representation. London: Phaidon.

Gombrich, E. H. (2000). Concerning 'The Science of Art': commentary on Ramachandran and
New analytic techniques will be necessary for understanding the whole physiological reaction, and open the opportunity for a converging approaches.

- An appropriate set of standardized measures and vocabulary for studying how non-professionals talk about and describe different aspect of the arts should be developed. Formalization of such categorization is fundamental to any meaningful integrative work.

- Future investigations should recognize that art is a dynamic cognitive process in which the definition of art is constantly changing in relation to its time. A more comprehensive approach should be used to explore the physiological characteristics and learning functions of this inherently chaotic modality.

\section{ACKNOWLEDGMENTS}

This research was supported by NSF/SLC Grants \#0824762 to C. W. Tyler and \#0846430 to L. T. Likova.
Hirstein. J. Conscious. Stud. 7, 17-27.

Hess, T. M., and Wallsten, S. M. (1987). Adult age differences in the perception and learning of artistic style categories. Psychol. Aging 2, 243-253.

Hetland, L., Winner, E., Veenema, S., and Sheridan, K. (2007). Studio Thinking: The Real Benefits of Visual Arts Education. New York: Teachers College Press.

Hollerman, J. R., Tremblay, L., and Schultz, W. (2000). Involvement of basal ganglia and orbitofrontal cortex in goal-directed behavior. Prog. Brain Res. 126, 193-215.

Kant, I. (1788). Critique of Practical Reason, trans. W. S. Pluhar. Toronto: Hackett Publishing Company.

Kanwisher, N., McDermott, J., and Chun, M. M. (1997). The fusiform face area: a module in human extrastriate cortex specialized for face perception. J. Neurosci. 17 4302-4311.

Kawabata, H., and Zeki, S. (2004). Neural correlates of beauty. J. Neurophysiol. 91, 1699-1705.

Kirk, A., and Kertesz, A. (1989). Hemispheric contributions to drawing. Neuropsychology 27, 881-886.

Kottlow, M., Praeg, E., Luethy, C., and Jancke, L. (2011). Artists' advance: decreased upper alpha power while drawing in artists compared with non-artists. Brain Topogr. 23, 392-402.

Kubovy, M. (1986). The Psychology of Perspective and Renaissance Art. Cambridge: Cambridge University Press.

Levitin, D. J. (2006). This Is Your Brain on Music: The Science of a Human Obsession. New York: Dutton/Penguin.
Likova, L. T. (2010a). "Drawing in the blind and the sighted as a probe of cortical reorganization," in Human Vision and Electronic Imaging XV: Proceedings of SPIE, Vol. 7527, eds B. E. Rogowitz and T. N. Pappas (Bellingham, WA: SPIE), $1-12$.

Likova, L. T. (2010b). The primary visual cortex as a modalityindependent 'screen' for working memory. J. Vis. 10, 776.

Likova, L. T. (2012a). Drawing enhances cross-modal memory plasticity in the human brain: a case study in a totally blind adult. Front. Hum. Neurosci. (in press).

Likova, L. T. (2012b). "The spatiotopic 'visual' cortex in the blind," in Human Vision Electronic Imaging: Proceedings of SPIE, eds B. E. Rogowitz, T. N. Pappas and H. de Ridder, Burlingame, 8291, 17.

Likova, L. T., and Tyler, C. W. (2008). Occipital network for figure/ground organization. Exp. Brain Res. 189, 257-267.

Livingstone, M. (2002). Vision and Art: The Biology of Seeing. New York: Harry N. Abrams.

McDougall, W. (1923). An Outline of Psychology. London: Methuen.

Murfee, E. (1995). Eloquent Evidence: Arts at the Core of Learning. Report by the President's Committee on the Arts and The Humanities. Washington, DC: National Assembly of State Arts Agencies.

Norcia, A. M., Candy, T. R., Pettet, M. W., Vildavski, V. Y., and Tyler, C. W. (2002). Temporal dynamics of the human response to symmetry. J. Vis. 2, 132-139.

Paulmann, S., Ott, D. V., and Kotz, S. A. (2011). Emotional speech perception unfolding in time: the role 
of the basal ganglia. PLOS ONE 6, e17694. doi: 10.1371/journal.pone. 0017694

Peterson, M. A. (1993). “The ambiguity of mental images: insights regarding the structure of shape memory and its function in creativity," in Imagery, Creativity, and Discovery: A Cognitive Perspective, eds B. RoskosEwoldsen, M. J. Itons-Peterson, and R. Anderson (Amsterdam: NorthHolland), 151-185.

Ramachandran, V. S., and Hirstein, W. (1999). The science of art: a neurological theory of aesthetic experience. J. Conscious. Stud. 6, 15-30.

Salimpoor, V. N., Benovoy, M., Larcher, K., Dagher, A., and Zatorre, R. J. (2011). Anatomically distinct dopamine release during anticipation and experience of peak emotion to music. Nat. Neurosci. 14, 257-262.

Sasaki, Y., Vanduffel, W., Knutsen, T., Tyler, C., and Tootell, R. (2005). Symmetry activates extrastriate visual cortex in human and nonhuman primates. Proc. Natl. Acad. Sci. U.S.A. 102, 3159-3163.

Seay, T. H. (2010). Feature: genetic dark matter. Sci. News 13, 18-28.
Smolucha, L., and Smolucha, F. (1985). A fifth Piagetian stage: the collaboration between analogical and logical thinking in artistic creativity. Vis. Arts Res. 11 91-99.

Solso, R. L. (2001). Brain activities in a skilled versus a novice artist: an fMRI study. Leonardo 34, 31-34.

Swindell, C. S., Holland, A. L., Fromm, D., and Greenhouse, J. B. (1988). Characteristics of recovery of drawing ability in left and right brain damaged patients. Brain Cogn. 7, 16-30.

Tononi, G. (2004). An information integration theory of consciousness. BMC Neurosci. 2, 42. doi:10.1186/1471-2202-5-42

Tucker, P., Rothwell, S., Armstrong, M., and McConaghy, N. (1982). Creativity, divergent, and allusive thinking in students and visual artists. Psychol. Med. 12, 835-841.

Tyler, C. W. (1994). Theoretical issues in symmetry perception. Spat. Vis. 8, 383-391.

Tyler, C. W. (1998). Painters centre one eye in portraits. Nature 392, 877-878.
Tyler, C. W. (2007). Some principles of spatial organization in art. Spat. Vis. 20, 509-530.

Tyler, C. W., Baseler, H. A., Kontsevich, L. L., Likova, L. T., Wade, A. R., and Wandell, B. A. (2005). Predominantly extraretinotopic cortical response to pattern symmetry. Neuroimage 24 306-314.

Tyler, C. W., Levitin, D., and Likova, L. T. (2009). NSF Workshop on Art, Creativity and Learning. Final Report. Washington, DC. Available at: http:// www.nsf.gov/sbe/slc/ACL_Report_ Final.pdf

Wallas, G. (1926). The Art of Thought. New York: Franklin Watts.

Winner, E., and Hetland, L. (2000). The arts and academic achievement: what the evidence shows. J. Aesthet. Educ. 34, 3-10.

Zaidel, D. W., and Cohen, J. A. (2005). The face, beauty, and symmetry: perceiving asymmetry in beautiful faces. Int. J. Neurosci. 15, 1165-1173.

Zeki, S. (1999). Inner Vision: An Exploration of Art and the Brain. Oxford: Oxford University Press.
Zeki, S. (2001). Essays on science and society. Artistic creativity and the brain. Science 293, 51-52.

Zeki, S. (2004). The neurology of ambiguity. Conscious. Cogn. 13, 173-196.

Conflict of Interest Statement: The authors declare that the research was conducted in the absence of any commercial or financial relationships that could be construed as a potential conflict of interest.

Received: 11 April 2011; paper pending published: 22 October 2011; accepted: 20 January 2012; published online: 08 February 2012

Citation: Tyler CW and Likova LT (2012) The role of the visual arts in enhancing the learning

process. Front. Hum. Neurosci. 6:8. doi: 10.3389/fnhum.2012.00008

Copyright (C) 2012 Tyler and Likova. This is an open-access article distributed under the terms of the Creative Commons Attribution Non Commercial License, which permits non-commercial use, distribution, and reproduction in other forums, provided the original authors and source are credited. 